(C) 1981. The Genetical Society of Great Britain

\title{
EVOLUTION OF ENVIRONMENTAL SEX DETERMINATION FROM GENOTYPIC SEX DETERMINATION
}

\author{
J. J. BULL \\ School of Biology, University of Sussex, Falmer, Brighton, Sussex BN1 9OG \\ Received 14.i.81
}

\section{SUMMARY}

\begin{abstract}
Environmental sex determination is a mechanism in which an individual's sex is decided after conception, according to its immediate environment. A previous theory proposed that environmental sex determination is adaptive in certain life histories by allowing control of sex in response to environmental effects on fitness. Although plausible, this theory did not explain how environmental sex determination evolves from an alternative mechanism. A model is presented here to describe the evolution of environmental sex determination from genotypic sex determination with male heterogamety $(X X \% / X Y$ ). As environmental sex determination evolves, both sexes become $X X$ and male heterogamety disappears. Except for certain types of $X / Y$ sex chromosome effects, a pre-existing genotypic mechanism of sex determination does not intrinsically interfere with the evolution of environmental sex determination, and the adaptation theory gives the correct conditions for its evolution. The widespread occurrence of $X X \% / X O{ }^{\star}$ sex chromosome systems in nematodes suggests that genotypic sex determination is possibly ancestral to environmental sex determination in this group, but convincing evidence is lacking.
\end{abstract}

\section{INTRODUCTION}

ENVIRONMENTAL sex determination is a mechanism in which zygotes differentiate as male or female depending largely upon environmental circumstances. It is not especially common in animals, but it is widespread, occurring in such diverse groups as reptiles (Bull, 1980), isopods, nematodes, and echiurids (Bacci, 1965). An additional feature is that the environmental determinant of sex differs among these groups: temperature determines sex in the reptiles, host size determines sex in the nematodes, and conspecific interactions determine sex in the others.

There are two schools of thought regarding the origin of environmental sex determination (ESD). One supposes that ESD is primitive, having been the first mechanism to produce two sexes, later being replaced by $X X / X Y$ genotypic mechanisms (Mittwoch, 1971, 1975; Ohno, 1967; Witschi, 1929). The other school supposes that ESD is an adaptation to particular life histories (Bacci, 1965; Charnov and Bull, 1977; described below). The views of these two schools are not incompatible, but if ESD is primitive and cannot arise from ordinary genotypic mechanisms such as male and female heterogamety, then any adaptive theory is limited in its applications. No empirical evidence has shown that ESD has arisen from genotypic sex determination (GSD), and no theory has demonstrated that the process is plausible. This paper describes a model for the transition from GSD (male heterogamety) to ESD. Sex determination in reptiles and nematodes is then reviewed briefly for evidence that ESD is derived from GSD. 


\section{ESD AS AN ADAPTATION: THEORIES}

Bacci (1965) suggested that ESD was an adaptation to parasitic existence, noting that nematodes, isopods, and echiurids with ESD are parasitic (if only in one sex). This theory did not otherwise explain why ESD evolves in such life histories. Charnov and Bull (1977) proposed more generally that ESD is favoured when two conditions occur in the life history: (i) early in life an individual enters a part of the environment which has a lasting effect on its fitness. The environment is patchy such that some patches enhance (or decrease) relative male fitness more than relative female fitness, and other patches do the opposite. (ii) Individuals have no control over which patch they enter. With random mating throughout the environment, these conditions select individuals which become female in patches where the greatest benefit (or least loss) is to femaleness and become male in patches where the greatest benefit is to maleness. GSD is selected against because it fixes sex at conception and thus often causes an individual to develop as the sex which benefits the least. With the exception of reptiles, species with ESD seem to have life histories with these characteristics (Charnov and Bull, 1977; Bull, 1980, 1981), thus accounting for Bacci's observations.

Theoretical studies have supported the hypothesis that sex determination in response to environmental conditions is favoured in the above life histories (Charnov, 1979; Bull, 1981; Bulmer and Bull, 1981). However, most of these models study the effect of an autosomal locus which modifies sex ratio, without regard for the segregation of genes from the ancestral sex determining mechanism. To more fully comprehend the evolution of ESD from GSD, it is desirable to consider the genotypic sex determining mechanism during the transition. The first step taken below is to describe the consequences of a combination of ESD and an $X X / X Y$ mechanism within a population; the second step is to describe how selection changes the frequency of ESD.

\section{A NEUTRAL PATH FROM $X X / X Y$ TO ESD}

Assume that as an ancestral state sex is determined according to a genotypic mechanism of male heterogamety, $X X+/ X Y \delta$, although what follows is equally applicable to female heterogamety with sexes reversed. $X$ and $Y$ may be considered as alleles at one locus or entire chromosomes (sex chromosomes). In order to introduce a component of ESD, some effect must begin to override ("reverse") sex determination so that occasional $X X$ males or $X Y$ females occur under certain environmental conditions. There are two somewhat different ways that this sex reversal might arise. As one possibility, all individuals may be nearly equally prone to sex reversal from some environmental extreme (e.g., in temperature or nutrition), but not all of the population experiences these extremes. Alternatively, a segregating gene may cause sex reversal in its carriers under certain environmental conditions, with non-carriers differentiating according to $X X / X Y$. The plausibility of these two causes of sex reversal is considered below. We proceed with the assumption of the first form of sex reversal, that all individuals are equally prone to sex reversal by an environmental factor. 
Having specified the nature of ESD, consider whether $X X, X Y$, and ESD may all be maintained in the population. For simplicity, assume that the environment causes sex reversal only in $X X:$ most $X X$ become female, but some become male. All $X Y$ are male. The frequency of $X Y$ then automatically changes to compensate for the $X X$ males, and the system evolves toward a sex ratio of $\frac{1}{2}$. In algebraic terms, let the starting frequency of $X Y$ among zygotes be $y$, of $X X$ be $1-y$, and a constant fraction $p$ of $X X$ become male. Fitness within a sex is the same for all genotypes. The sex ratio is $M=y+p(1-y)$, and the frequency of $X Y$ zygotes in the next generation is $y^{\prime}=y / 2 M$. Therefore, $X Y$ increases if $M<\frac{1}{2}$, decreases if $M>\frac{1}{2}$, and the sex ratio in the next generation $\left(M^{\prime}\right)$ increases or decreases with $X Y$. More formally,

$$
\left(M-\frac{1}{2}\right)^{\prime}=\frac{p}{M}\left(M-\frac{1}{2}\right)
$$

and

$$
\left(y-\frac{\frac{1}{2}-p}{1-p}\right)^{\prime}=\frac{p}{M}\left(y-\frac{\frac{1}{2}-p}{1-p}\right),
$$

where $M=y+p(1-y)$, and $p=$ constant. Thus the sex ratio evolves toward $\frac{1}{2}$, with the deviation reduced each generation to $p / M$ of its former value (if $p \leqq \frac{1}{2}$ ). From (2) the change in sex ratio coincides with a change in the frequency of $X Y$. If instead $p>\frac{1}{2}$, the frequency of $X Y$ is diminished toward zero, and the sex ratio becomes $p$.

This result shows that when there is a combination of $X X \% / X Y \delta$ sex determination as well as some environmental sex determining factor $\left(p \leqq \frac{1}{2}\right)$, the sex ratio evolves to $\frac{1}{2}$ by change in the frequency of $X Y$. This conclusion also holds if $p$ is controlled by some genetic factor, although the dynamics above do not apply because $p$ and $y$ both change. It can further be shown that there is no tendency for selection to change the value of $p$ when the sex ratio has equilibrated. There is thus a continuum of selectively neutral equilibria from strict $X X q / X Y \delta$ sex determination $(p=0)$ to complete environmental sex determination $\left(p=\frac{1}{2}\right)$. The selective neutrality of this path necessarily applies only if all genotypes within a sex are equally fit, which may rarely be true in nature. The result is important to a wider realm of circumstances, however, because it shows that there is no intrinsic disadvantage of evolving from male heterogamety to ESD or the reverse. Thus, even a slight fitness difference which favours ESD may be sufficient to select for the loss of GSD.

The neutral path between male heterogamety and ESD also exists when the environment causes femaleness of some $X Y$ (and $Y Y$ ) zygotes, again assuming that fitness is equal for all genotypes within a sex. In this case the equilibrium frequency of $Y$ increases as the environment is more female determining, and complete ESD is achieved with both sexes as $Y Y$. This path may not of ten be feasible, because $Y Y$ is presumably lethal in species where the $Y$ is a differentiated sex chromosome (see Discussion).

\section{SELECTION FOR ESD}

Charnov and Bull (1977) argue that ESD is selected if fitness correlates with some environmental parameter, differentially for males and females. 
The above model is inadequate to deal with this hypothesis for two reasons: (i) fitness is held constant within each sex, and (ii) the level of environmental sex determination (of $X X$ ) is not allowed to evolve. An extension of the above model is offered in order to encompass these properties. As above, zygotes are either $X X$ or $X Y$, and some $X X$ as well as all $X Y$ develop as male.

\section{(i) Environmental effects on fitness}

The Charnov-Bull model proposes that ESD evolves in response to environmental variables which influence the individual's expected fitness. As an example, it is plausible for many species that an individual's expected reproductive success depends upon its size, this often being limited by nutrition early in life. Nutrition may of ten vary among individuals, not because of genetic differences, but merely because of chance. Thus size, and hence fitness, would have a large environmental component. In the model, let $T$ be an environmental variable affecting fitness. Each zygote is born at a random value of $T=t$ and has fitness $1+\alpha t$ as male, or $1+\beta t$ as female. The mean of $T$ is taken as zero and the variance of $T$ is assumed small enough that negative fitnesses accrue to a negligible fraction of the population. The linear dependence of fitness with $T$ is assumed for mathematical convenience; presumably the qualitative form of the results is not sensitive to strict linearity of the fitness function.

\section{(ii) Selecting the level of ESD}

In order that the level of ESD may evolve, $X X$ zygotes must show heritable differences in their tendency to be male. Furthermore, sex determination must also depend upon the environmental effect, $T$, if it is to be considered ESD. A rather artificial model is offered to incorporate these properties, though the model is of a type widely used in quantitative genetics (Falconer, 1960; Bulmer, 1980). Let $S$ be the inherited variable of sex determination in $X X$ zygotes. Each zygote is born with a value of $S$, determined by genetic effects at many loci plus an environmental effect, with some heritability due to the genetic transmission (i.e., $S$ is a quantitative genetic character). Sex is determined according to whether $S-T$ is greater or less than a "threshold", taken without loss of generality as zero. Thus, an $X X$ zygote becomes male if $S-T>0$, female if $S-T<0$ (fig. 1; Bulmer and Bull, 1981). All $X Y$ zygotes are male despite their values of $S-T$. Once the distributions of $S$ and $T$ are specified, the effect of selection on $S$, and thus on the level of ESD, may be calculated.

A brief digression here provides some motivation for the form of this model. Models of this sort are commonly used in the literature of quantitative genetics for the study of discontinuous characters, such as disease or meristic characters (Wright, 1968, pp. 266, 292; Falconer, 1960; Bulmer, 1980). Bulmer and Bull (1981) have recently extended this type of model to include sex determination. There is no direct way of observing whether sex is determined in exactly this fashion, but many properties of the model can be verified from studies of populations. In particular, the heritability of $S$ can be measured, and its magnitude indicates how readily differences in $X X$ maleness can be selected (Appendix; Bulmer and Bull, 1981; Bull, 


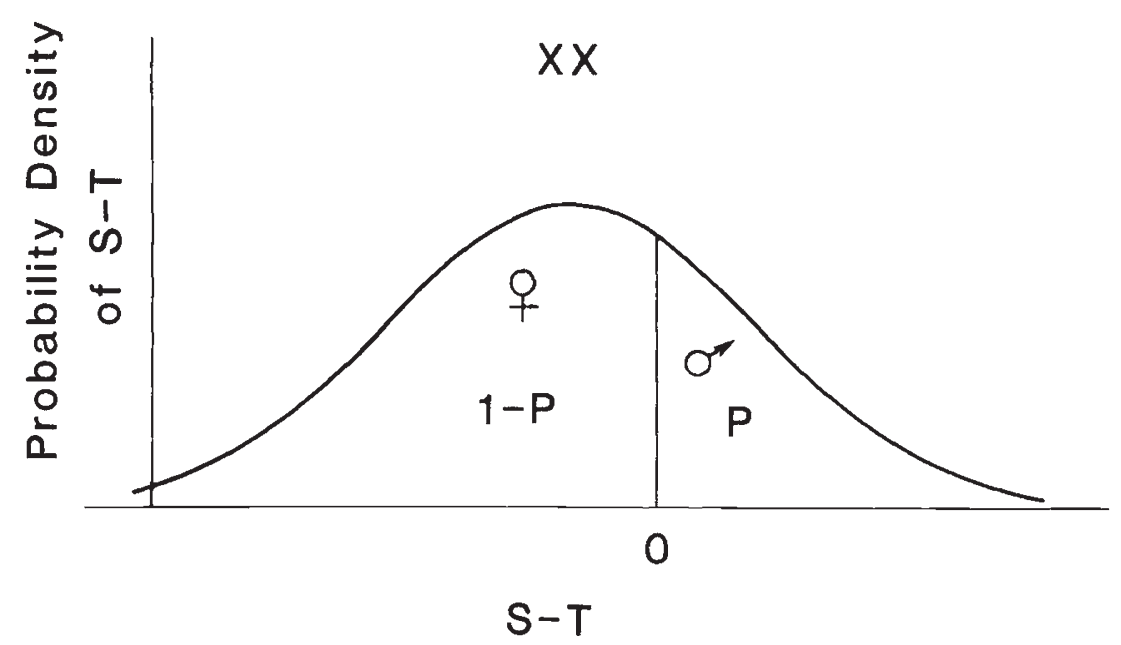

FIG. 1.-Model of environmental sex determination in $X X$ zygotes. $T$ is an environmental variable (e.g., temperature) and $S$ is a zygotic phenotype determined partly by inheritance. $X X$ is male if $S-T>0$, female if $S-T<0$. $P$ is the proportion of $X X$ which are male.

Vogt, and Bulmer, 1982). It is supposed that the qualitative results of this model reflect a more general nature of selection on ESD than that specific to this formulation.

The evolution of ESD depends upon the change in the mean of $S(\bar{S})$. As $\bar{S}$ increases, the frequency of $X X$ males $(S-T>0)$ also increases, whereas the (equilibrium) frequency of $X Y$ decreases. Assuming that $S$ and $T$ are distributed normally, the Appendix shows that the path of neutral equilibria between ESD and GSD exists only if $\alpha=\beta$, when fitness in both sexes changes identically with $T$. For $\alpha \neq \beta$, numerical iteration and analytical results (Appendix) suggest that ESD evolves if $\beta>\alpha$, GSD evolves if $\alpha>\beta$. ESD evolves with the complete loss of $X Y$ males in the population, and both sexes become $X X$. Thus all traces of male heterogamety vanish.

It is surprising that $\alpha>\beta$ selects for GSD rather than some other kind of ESD. This is an artifact of the model. $\alpha>\beta$ selects for GSD because the model only allows $X X$ males for $S-T>0$. If $X X$ is male for $S-T<0$, female for $S-T>0, \alpha>\beta$ selects for $\operatorname{ESD}(\beta>\alpha$, against). Also, ESD would be likely to evolve if $X Y$ and $Y Y$ are subject to sex reversal rather than $X X$. These constraints imposed by the model are artificial, but similar constraints may conceivably operate in nature.

These results support the hypothesis of Charnov and Bull (1977). ESD evolves if $\beta>\alpha$, implying that females benefit more from high $T$ than males. Since an $X X$ individual is more likely to be female $(S-T<0)$ if it encounters a high temperature than a low one, females on average will have higher $T$ values than males. Thus, females develop in patches which offer the greatest fitness gain (or least loss) to femaleness, and similarly for males. The Charnov-Bull argument also predicts that $\alpha>\beta$ selects ESD with males at high temperatures and females at low ones, and as stated above, this result follows in the analogous model which allows $X X$ males to develop at higher temperatures than females. The model shows 
that there is a plausible evolutionary path from an $X X+/ X Y \hat{\delta}$ system to ESD, so ESD need not be regarded as primitive on theoretical grounds.

\section{Discussion}

Charnov and Bull (1977) proposed that environmental sex determination is advantageous in certain life histories because it allows the embryo to adjust its sex according to environmental effects on fitness. Genotypic sex determination fixes sex at conception and therefore does not allow this control. The hypothesis that selection favours sex ratio adjustment in response to the environment originates with Trivers and Willard (1973), and has since been supported by theoretical studies (Charnov, 1979; Charnov et al., 1978; Bull, 1981; Bulmer and Bull, 1981) and empirical observations (Charnov and Bull, 1977; Charnov et al., 1978, 1981). The argument of Charnov and Bull goes one step further, to suppose that if selection favours sex ratio control, then selection necessarily favours a sex determining mechanism which allows this control. This second part of the argument has remained largely conjectural, because theoretical studies have not usually investigated the evolution of the sex determining mechanism. Recently, Bulmer and Bull (1981) have shown that the evolution of ESD from polygenic sex determination is governed by selection according to the Charnov-Bull argument. This paper investigates another possible path from GSD to ESD, starting with an $X X / X Y$ ancestor, and shows that selection for ESD is also given by the Charnov-Bull argument. Because of the ubiquity of male and female heterogamety in animals, there are many opportunities for the evolution of ESD along this path.

Whether ESD has actually evolved from male or female heterogamety may be difficult to establish, although there are several lines of evidence which may be used to investigate this question.

(1) Simple phylogenetic considerations may indicate the probable ancestral sex determining mechanism. For example, a single instance of ESD amid a lineage otherwise of male or female heterogamety may support a hypothesis that GSD is ancestral, if many independent origins of GSD are required to suppose a different ancestor. Some additional evidence is desired to show that all mechanisms of GSD are themselves probably derived from one common ancestor.

(2) Populations polymorphic for GSD and ESD may be segregating a highly differentiated $Y$ chromosome if they are derived from a long-established $X X / X Y$ mechanism. The evolution of distinct cytological characteristics of the $Y$ is apparently a slow process (Lucchesi, 1978; Charlesworth, 1978), so that if the $X Y$ system has evolved subsequent to ESD, the $Y$ is not likely to manifest these unusual characteristics.

(3) Populations with ESD should be homozygous for the $X$ or $Y$ if they are descended from an $X X / X Y$ system. This will be difficult to ascertain unless the ancestral $X$ and $Y$ are distinct sex chromosomes, and in this case ESD is only likely to evolve as $X X$, because $Y Y$ is not likely to be viable. $X$ chromosomes are often unusual in meiotic behavior, timing of replication and condensation, and in affecting sex determination when aneuploid $(X O, X X X)$ (White, 1973 ), and these properties may be retained after ESD evolves. 
Thus although the model does not require that $X$ and $Y$ be distinct sex chromosomes, to demonstrate that ESD is derived from male or female heterogamety is most feasible if differentiated sex chromosomes characterise the ancestors.

Reptiles and nematodes are the only groups with sufficient data on sex determining mechanisms to permit investigation of the origins of ESD. Both ESD and sex chromosomes are widespread in reptiles, occurring together in two orders (Bull, 1980). In nematodes, $X O$ sex chromosome systems are widespread, and ESD is found in two parasitic groups distantly related to each other (White, 1973; Chitwood and Chitwood, 1974). No intermediates between GSD and ESD are recognised in nematodes or reptiles, so any demonstration that $\mathrm{ESD}$ is derived from $X X / X Y$ must at present be based on interspecific comparisons.

Cytological evidence from reptiles suggests that sex chromosome heteromorphism is recently evolved (Bull, 1980). The only group in which there seems to be a remote ancestry of heteromorphic sex chromosomes is the suborder of snakes (Ohno, 1967), and there is yet no evidence of ESD in snakes. Otherwise, sex chromosome heteromorphism has arisen sporadically in reptiles, and the $X$ of one species is not necessarily recognisable as the $X$ in close relatives. It is therefore difficult to trace the ancestry of male and female heterogamety in reptiles. It is altogether possible that ESD is the ancestral mechanism for reptiles, although the case for GSD as the ancestor is only slightly weaker (Bull, 1980).

Nematodes have a remote ancestry of sex chromosomes, and for this reason ESD may be suspected as a derivative of GSD. $X X \% / X O \delta$ systems are widespread and characterise many or the majority of nematodes with separate sexes; other sex chromosome systems $(X X / X Y$, multiple $X$ 's) are apparently derivations of the simpler $X O$ condition (White, 1973; Triantaphyllou, 1973). A remote, common ancestry of these different mechanisms is suggested because more than one independent origin of $X O$ seems unlikely: loss of the $Y$ in animals is apparently a lengthy process of infrequent occurrence (e.g., White, 1973).

However, although it is plausible that ESD is derived from $X X / X O$ in nematodes, there is little basis for a rigorous assessment of this hypothesis. Two lines of investigation may eventually provide discriminating evidence. (i) Both sexes of species with ESD which have been studied cytologically have the same chromosome number (Triantaphyllou, 1973), as expected if the environment determines sex. If ESD is derived from $X X / X O$, then these species are likely to be $X X$ in both sexes. Occasional $X O$ males might therefore arise, but as yet none has been reported. It is interesting that several hermaphroditic nematodes produce occasional $X O$ males, indicating that hermaphroditism evolved subsequent to $X X q / X O{ }^{\circ}$. In the hermaphrodites, the males are a novelty and are consequently studied. In species with ESD, $X O$ males would not necessarily be morphologically distinguishable from $X X$ males, and they might therefore escape detection in all but the most exhaustive cytological studies. (ii) If ESD is recently derived from $X X / X O$, species with ESD might have $X X / X O$ close relatives. The evolution of $X O$ is not expected in species with a recent ancestry of ESD, so there would be little doubt that ESD was derived. The present disarray in nematode taxonomy precludes drawing any firm conclusion that 
ESD and $X X q / X O \delta$ occur within the same order; by some classifications they do, but by others they do not (Chitwood and Chitwood, 1974; Rothschild, 1965). One species which may be $X X / X O$ (Seinura tenuicaudata) is considered by some to be a member of the same order as plant parasites with ESD, but there is some doubt that the species is in fact $X O$ (Hechler, 1963).

The transition from $X X / X Y$ to ESD as presented here is not the only path by which ESD need evolve. The selective forces which favor ESD may often be the same (i.e., the Charnov-Bull model of sex ratio adjustment), but there is no reason to suppose that the sex determining mechanism in the transition is always as depicted here. The process described in the model is plausible, however. Environmental effects such as extreme temperature, egg overripeness, and hormones cause sex reversal in amphibians (reviewed in Bull, 1980). Some of these effects may even occur in nature, because $X Y$ females and their $Y Y$ progeny have been observed in some wild populations with male heterogamety (Kawamura and Nishioka, 1977). It also seems likely that there would be at least slight, heritable differences of individual response to these factors, corresponding to $S$ in the model. An alternative possibility considered above, that ESD arises from environmental-sensitive mutations with major effect, has less support. Mutations are known which cause $X X$ maleness in flies and hermaphroditic nematodes (Wagoner, 1969; Baker and Ridge, 1980; Hodgkin and Brenner, 1977), but there is no evidence as yet that any of these are environmentalsensitives.

The evolution of ESD from $X X / X Y$ is constrained so that the fitness of $X X$ males is not intrinsically less than that of $X Y$ males (or in the alternative pathway, $X X, X Y$ and $Y Y$ individuals must be equally fit within a sex). This may usually be true of species with undifferentiated sex chromosomes, because sex reversals are apparently normal and $Y Y$ is even fully fertile in some amphibians and fish which lack cytological heterogamety (Bull, 1980; Yamamoto, 1969). In species with heteromorphic sex chromosomes, the $Y$ is often degenerate or absent so that $Y Y$ $(O O)$ is inviable (Lucchesi, 1978; Charlesworth, 1978). The only path to ESD could be through $X X$ maleness. And although $X X$ males are likely to be viable in species with differentiated sex chromosomes, they will not necessarily be fertile: in Drosophila melanogaster, the $Y$ chromosome contains genes vital to sperm production, so that $X X$ males (without a $Y$ ) are necessarily sterile (Hess and Meyer, 1968). Another possible complication is that ESD may cause intersexual types of low fitness, unless there is a physiological mechanism to prevent individuals from remaining part male and part female. In view of these possible complications, the evolution of ESD from male or female heterogamety should not be regarded as always feasible. If, however, individuals whose sex is determined by the environment are not intrinsically inferior to the others, then the evolution of ESD is governed by the selective forces originally proposed by Charnov and Bull (1977).

\section{REFERENCES}

BACCI, G. 1965. Sex Determination. Pergamon Press, Oxford.

BAKER, B. S., AND RIDGE, K, 1980. Sex and the single cell. I. On the action of major loci affecting sex determination in Drosophila melanogaster. Genetics, 94, 383-423. 
BULl, J, J. 1980. Sex determination in reptiles, Quart, Rev. Biol., 55, 3-21.

BulL, J. J. 1981. Sex ratio evolution when fitness varies. Heredity, 46, 9-26.

BUll, J. J., VOGT, R. C., AND BULMER, M. G. 1982. Heritability of sex ratio in turtles with environmental sex determination. MS (submitted).

BUlmer, M. G. 1980. The Mathematical Theory of Quantitative Genetics. Oxford University Press, Oxford.

BUlmer, M. G., AND BULl, J. J. 1981. Models of polygenic sex determination and sex ratio evolution. Evolution (in press).

CHARLESWORTH, B. 1978. Model for evolution of Y chromosomes and dosage compensation. Proc. Nat. Acad. Sci. USA, 75, 5618-5622.

CHARNOV, E. L. 1979. The genetical evolution of patterns of sexuality: Darwinian fitness. Am. Nat., 113, 465-480.

CHARNOV, E. L., AND BULL, J. J. 1977. When is sex environmentally determined? Nature, $266,828-830$.

CHARNOV, E. L., GOTSHALL, D., AND ROBINSON, J, 1978. Sex ratio: adaptive response to population fluctuations in pandalid shrimp. Science, 200, 204-206.

CHARNOV, E. L., LOS-DEN HARTOGH, R. L., JONES, T., AND VAN DEN ASSEM, J. 1981. Sex ratio evolution in a patchy environment. Nature (in press).

CHITwood, B. G., AND CHITwOOD, M. G. 1974. An Introduction to Nematology (revised). University Park Press, Baltimore.

FALCONER, D. S. 1960. Introduction to Quantitative Genetics. Ronald Press Co., New York.

HECHLER, H. C. 1963. Description, developmental biology, and feeding habits of Seinura tenuicaudata (De Man) J. B. Goodey, 1960 (Nematoda: Aphelenchoididae), a nematode predator. Proc. Helm. Soc., 30, 182-195.

HESS, O., AND MEYER. G, F, 1968. Genetic activities of the Y chromosome in Drosophila during spermatogenesis. Adv. Genet., 14, 171-223.

HODGKIN, J, A., AND BRENNER, S. 1977. Mutations causing transformation of sexual phenotype in the nematode Caenorhabditis elegans. Genetics, 86, 275-287.

KAWAMURA, T. AND NISHIOKA, M. 1977. Aspects of the reproductive biology of Japanese anurans. In The Reproductive Biology of Amphibians, eds. D. H. Taylor and S. Guttman, Plenum, New York.

LUCCHESI, J. C. 1978. Gene dosage compensation and the evolution of sex chromosomes. Science, 202, 711-716.

MITTWOCH, U. 1971. Sex determination in birds and mammals. Nature, 231, 432-434.

MITTWOCH, U. 1975. Chromosomes and sex differentiation. In Intersexuality in the Animal Kingdom, ed. R. Reinboth. Springer-Verlag, Berlin.

OHNO, S. 1967. Sex Chromosomes and Sex-Linked Genes. Springer-Verlag, Berlin.

ROTHSCHILD, L. 1965. A Classification of Living Animals. Longmans, Green and Co. Ltd., London.

TRIANTAPHYLLOU, A. C. 1973. Environmental sex determination of nematodes in relation to pest management. Ann. Rev. Phytopath., 11,441-462.

TRIVERS, R. L., AND WILLARD, D. E. 1973. Natural selection of parental ability to vary the sex ratio of offspring. Science, 179, 90-92.

WAGONER, D. E. 1969. Presence of male determining factors found on three autosomes in the housefly, Musca domestica. Nature, 223, 187-188.

WHITE, M. J. D. 1973. Animal Cytology and Evolution. 3rd ed. Cambridge University Press, Cambridge.

WITSCHI, E. 1929. Studies on sex differentiation and sex determination in amphibians. III. Rudimentary hermaphroditism and $\mathrm{Y}$ chromosome in Rana temporaria. J. Exp. Zool., 54, 157-223.

WRIGHT, s. 1968. Evolution and the Genetics of Populations. Vol. 1. University of Chicago Press, Chicago.

y Amamoto, T. 1969. Sex differentiation. In Fish Physiology, eds. W. S. Hoar and D. J. Randall, Vol. 3. Academic Press, New York.

\section{APPENDIX}

The model of environmental sex determination in the text is similar to that of Bulmer and Bull (1981), and many of the results required here are derived in their paper. 
From the text, $T$ is an environmental variable affecting fitness of all zygotes and affecting sex in $X X$ zygotes. $S$ is a heritable phenotype which affects the response to $T$ in being an $X X$ male:

$$
\begin{array}{ll}
X X \text { is male if } \quad & S-T>0, \\
X X \text { is female if } & S-T<0 .
\end{array}
$$

$T$ is chosen at random for each zygote, but $S$ is determined at least partly by inheritance. In $X Y$ zygotes this phenotypic character is labelled $Q$ rather than $S$ since its distribution in $X Y$ is not generally the same as in $X X . X Y$ is always male, however. At $T=t$, fitness is

$$
\begin{array}{ll}
1+\alpha t & \text { for males, } \\
1+\beta t & \text { for females. }
\end{array}
$$

For mathematical tractability, $S, Q$, and $T$ are assumed to have normal distributions:

$$
\begin{aligned}
& S \sim n\left(\bar{S}, \sigma_{s}^{2}\right)=f(s), \\
& Q \sim n\left(\vec{Q}, \theta^{2}\right)=h(q), \\
& T \sim n\left(0, \omega^{2}\right)=g(t) .
\end{aligned}
$$

The frequency of $X Y$ zygotes is denoted $y$, as in the text. From this it follows that $P^{*}$, the fitness times the fraction of $X X$ which are male, is

$$
P^{*} \equiv \int_{-\infty}^{\infty}(1+\alpha t) g(t) \int_{t}^{\infty} f(s) d s d t .
$$

From Bulmer and Bull (1981, Appendix) this is

$$
P^{*}=P-\frac{\alpha \omega^{2}}{\sigma} \phi\left(\frac{\bar{S}}{\sigma}\right)
$$

where $\sigma^{2}=\sigma_{s}^{2}+\omega^{2}, \phi(x)$ is the standard normal density function evaluated at $x$, and $P$ is the proportion of males $\int_{-\infty}^{\infty} g(t) \int_{t}^{\infty} f(s) d s d t=\operatorname{Pr}\{S-T>0\}=$ $\Phi(\bar{S} / \sigma)$.

\section{(i) Evolution of $\mathrm{XY}$}

$$
y^{\prime}=\frac{y}{2 M}
$$

where $M=y+P^{*}(1-y)$. This can be rewritten as

$$
y^{\prime}-\frac{\frac{1}{2}-P^{*}}{1-P^{*}}=\frac{P^{*}}{M}\left(y-\frac{\frac{1}{2}-P^{*}}{1-P^{*}}\right) .
$$

(ii) Evolution of $\mathrm{P}$ and $\overline{\mathrm{S}}$

From Bulmer and Bull (1981), the mean value of $S$ in $X X$ males is

$$
\frac{\int_{-\infty}^{\infty}(1+\alpha t) g(t) \int_{t}^{\infty} s f(s) d s d t}{\int_{-\infty}^{\infty}(1+\alpha t) g(t) \int_{t}^{\infty} f(s) d s d t}=\bar{S}+k A,
$$


in which $k=\left(\sigma_{s}^{2} / \sigma\right) \phi(\bar{S} / \sigma)$, and $A=\left(1+\left(\alpha \omega^{2} / \sigma^{2}\right) \bar{S}\right) / P^{*}$. The mean value of $S$ in $X X$ females is

$$
\frac{\int_{-\infty}^{\infty}(1+\beta t) g(t) \int_{-\infty}^{t} s f(s) d s d t}{\int_{-\infty}^{\infty}(1+\beta t) g(t) \int_{-\infty}^{t} f(s) d s d t}=\bar{S}-k C,
$$

in which $k$ is as above and

$$
C=\left(1+\frac{\beta \omega^{2}}{\sigma^{2}} \bar{S}\right) /\left\{1-P+\frac{\beta \omega^{2}}{\sigma} \phi\left(\frac{\bar{S}}{\sigma}\right)\right\}
$$

(Bulmer and Bull 1981, Appendix). Therefore, the average transmitted value of $S$ from

$$
\begin{array}{cl}
X X \text { males is } & S_{1}=\bar{S}+h^{2} k A, \\
X X \text { females is } & S_{2}=\bar{S}-h^{2} k C,
\end{array}
$$

and $X Y$ males is $\bar{Q}$, where $h^{2}$ is the heritability of $S$.

$X X$ zygotes come from two types of parental matings,

$$
\begin{array}{cccc}
\text { frequency } & \text { parents } & & \text { zygotes } \\
\frac{P^{*}(1-y)}{M} & \frac{\delta}{X X} \cdot \frac{\wp}{X X} & \rightarrow & X X \\
\frac{y}{M} & X Y \cdot X X & \rightarrow & \frac{1}{2} X X+\frac{1}{2} X Y
\end{array}
$$

The mating of $X X \delta . X X$ q yields a mean value of $S$ in zygotes of $\left(S_{1}+S_{2}\right) / 2$, and $X Y \vec{\delta} . X X$ yields a mean value of $\left(\vec{Q}+S_{2}\right) / 2$. Thus,

$$
\begin{aligned}
& \bar{S}^{\prime}=\frac{P^{*}(1-y)\left(S_{1}+S_{2}\right)+\frac{1}{2} y\left(\bar{Q}+S_{2}\right)}{2 P^{*}(1-y)+y}, \\
& \bar{Q}^{\prime}=\frac{1}{2}\left(S_{2}+\bar{Q}\right)=\frac{1}{2}(\bar{Q}+\bar{S})-\frac{1}{2} h^{2} k C .
\end{aligned}
$$

\section{(iii) Evaluation of equilibria}

From (A.3b), the frequency of $X Y$ is stationary if $y=\left(\frac{1}{2}-P^{*}\right) /\left(1-P^{*}\right)$. Substituting this value into (A.6),

$$
\Delta \bar{S}=\bar{S}^{\prime}-\bar{S}=(\bar{S}-\bar{Q})\left(P^{*}-\frac{1}{2}\right)+h^{2} k\left\{P^{*} A-\frac{1}{2} C\right\} .
$$

Differencing (A.7) and (A.8),

$$
\bar{S}^{\prime}-\bar{Q}^{\prime}=P^{*}(\bar{S}-\bar{Q})+h^{2} k P^{*} A,
$$

hence at equilibrium,

$$
\bar{S}-\bar{Q}=\frac{P^{*}}{1-P^{*}} h^{2} k A .
$$


Substituting this into (A.8),

$$
\Delta \bar{S}=\frac{1}{2} h^{2} k\left(\frac{A P^{*}}{1-P^{*}}-C\right),
$$

or

$$
\Delta \bar{S}=\frac{1}{2} h^{2} k \frac{\left\{\phi\left(\frac{\bar{S}}{\sigma}\right)-\frac{\bar{S}}{\sigma}(1-P)\right\} \frac{\omega^{2}}{\sigma}(\beta-\alpha)}{\left\{1-P+\frac{\alpha \omega^{2}}{\sigma} \phi\left(\frac{\tilde{S}}{\sigma}\right)\right\}\left\{1-P+\frac{\beta \omega^{2}}{\sigma}\left(\frac{\bar{S}}{\sigma}\right)\right\}}
$$

$\Delta \bar{Q}$ is equal to $\Delta \bar{S}$ in (A.11), assuming (A.10).

The infinite path of neutral equilibria from GSD to ESD exists in general only for $\alpha=\beta$. If $\alpha \neq \beta$ two equilibria are guaranteed, $X Y$ males absent $(y=0)$ or $X X$ males absent $\left(y=\frac{1}{2}\right.$; strictly true only in the limit $\left.\bar{S}=-\infty\right)$. Perhaps the term $\{\phi(\bar{S} / \sigma)-\bar{S}(1-P) / \sigma\}$ admits other equilibria for $\alpha \neq \beta$, but this is possible only if $\bar{S}>0\left(P>\frac{1}{2}\right.$, with $X Y$ rare $)$. With $|\alpha+\beta|$ sufficiently small, however, $\bar{S} / \sigma$ can be guaranteed small at equilibrium, and no other roots exist.

(iv) Transition between GSD and ESD: $\alpha \neq \beta$

Equation (A.11b) shows that starting from equilibrium for $y$ with respect to $P^{*}$ and $\bar{S}-\bar{Q}$, the frequency of $X X$ males increases if $\beta>\alpha$, decreases if $\alpha>\beta$. In the next generation $y$ and $\bar{S}-\bar{Q}$ are not at equilibrium, and it is difficult to predict how they change thereafter. It would be surprising if in the long term $\bar{S}$ did not increase generally for $\beta>\alpha$, and decrease for $\alpha>\beta$, but verification of this is needed. Numerical iteration of (A.3a), (A.6), and (A.7) was performed to further examine the effect of $\beta>\alpha$, $\alpha=\beta$, and $\alpha>\beta$ on the change in $\bar{S}$. For $\sigma_{s}^{2}=\omega^{2}=1, h^{2}=0.5,1$, and various initial conditions, selection ultimately increased the frequency of ESD for $\beta>\alpha$, decreased its frequency if $\alpha>\beta$, and equilibrated at polymorphism if $\alpha=\beta$, consistent with these limited analytical results. $|\alpha-\beta|$ in these trials was often as small as 0.01 . 